\title{
Identification of clinical factors associated with severe dengue among Thai adults: a prospective study
}

\author{
Vipa Thanachartwet ${ }^{1}$, Nittha Oer-areemitr ${ }^{2}$, Supat Chamnanchanunt ${ }^{1}$, Duangjai Sahassananda ${ }^{3}$, \\ Akanitt Jittmittraphap ${ }^{4}$, Plengsakoon Suwannakudtt, Varunee Desakorn ${ }^{1}$ and Anan Wattanathum ${ }^{2}$
}

\begin{abstract}
Background: Dengue is the most common mosquito-borne viral disease in humans. Recently, there has been an epidemic shift of dengue from mainly affecting children to affecting more adults with increased severity. However, clinical factors associated with severe dengue in adults have varied widely between studies. We aimed to identify the clinical factors associated with the development of severe dengue according to the World Health Organization (WHO)'s 2009 definition.
\end{abstract}

Methods: We conducted a prospective study of adults with dengue admitted to the Hospital for Tropical Diseases in Bangkok, Thailand, from October 2012 to December 2014. Univariate and stepwise multivariate logistic regression analyses were performed.

Results: Of the 153 hospitalized patients with confirmed dengue viral infections, 132 (86.3\%) patients had non-severe dengue including dengue without warning signs (7 patients, $5.3 \%)$ and dengue with warning signs $(125,94.7 \%)$. The rest $(21,13.7 \%)$ had severe dengue including severe plasma leakage $(16,76.2 \%)$, severe organ involvement $(16,76.2 \%)$, and severe clinical bleeding $(8,38.1 \%)$. Using stepwise multivariate logistic regression, clinical factors identified as independently associated with the development of severe dengue were: (1) being $>40$ years old (odds ratio [OR]: 5.215, 95 \% confidence interval [Cl]: 1.538-17.689), (2) having persistent vomiting (OR: 4.817, Cl: 1.375-16.873), (3) having $>300$ cells per $\mu \mathrm{L}$ of absolute atypical lymphocytes (OR: 3.163, Cl: 1.017-9.834), and (4) having lactate levels $\geq 2.0 \mathrm{mmol} / \mathrm{L}$ (OR: 7.340, Cl: 2.334-23.087). In addition, increases in lactate and absolute atypical lymphocyte levels corresponded with severe dengue $(p<0.05)$.

Conclusions: Our study identified several clinical factors independently associated with the development of severe dengue among hospitalized adults with dengue. This can aid in the early recognition and prompt management of at-risk patients to reduce morbidity and mortality.

Keywords: Dengue fever, Lactate, Thailand, Predictive factors

\section{Background}

Dengue is caused by four dengue virus serotypes (DENV 1-4) and it is the most rapidly spreading mosquito-borne viral disease in humans [1]. Annually, approximately 50-100,000 000 people contract dengue viral infections worldwide and an estimated 500,000 people with dengue hemorrhagic fever (DHF) require

\footnotetext{
*Correspondence: vipa.tha@mahidol.edu

'Department of Clinical Tropical Medicine, Faculty of Tropical Medicine, Mahidol University, 420/6 Rajvithi Road, Bangkok 10400, Thailand Full list of author information is available at the end of the article
}

hospitalization [2]. The mortality rate of dengue is approximately 20,000 deaths per year with a morbidity of 264 disability-adjusted life years per million people per year [1]. During the past decades, the emergence of dengue has been reported throughout the world with a 30-fold increase in global incidence and severity. Approximately $75 \%$ of the global populations exposed to dengue reside in the Asia-Pacific region [1].

Previous reports have shown an epidemic shift of dengue from mainly affecting children to now affecting more adults [2-4]. Children and adults with dengue 
differ in both clinical manifestations and laboratory findings $[5,6]$, and the incidence of "severe dengue" according to the WHO's 2009 definition has increased among adults [2, 7]. In 2009, the WHO introduced a new dengue case definition to emphasize clinical management and to increase the sensitivity and specificity of severedengue diagnosis [8]. A recent systemic review of the application of the WHO's 2009 definition showed a sensitivity of $59-98 \%$ and a specificity of $41-99 \%$ for identifying severe dengue [9].

In Thailand, the number of adults with dengue has increased dramatically in recent decades $[1,4]$. According to the WHO's 2009 definition, approximately $27.9 \%$ of hospitalized Thai adults with dengue had severe dengue from 2006 to 2010 [10]. Although several studies have been conducted, the clinical factors associated with severe dengue in adults have varied widely due to (1) the differences in applying the definitions of severe dengue, by using either the WHO's 1997 definition or the WHO's 2009 definition, (2) differences in the study designs, and (3) differences in the data analyses [10-14]. Thus, these studies have reported widely varying results of the clinical factors associated with severe dengue, including having secondary dengue infections, age $>37$ years, male gender, presence of intense asthenia, presence of abdominal pain, presence of cough, presence of bleeding, a mean arterial pressure (MAP) less than $80 \mathrm{mmHg}$, an increase in hematocrit levels to more than $2 \%$ over the reference range (adjusted for gender), low lymphocyte level, higher aspartate aminotransferase (AST) or alanine aminotransferase (ALT) levels by more than 3 times, and low total protein level [10-14]. Currently, the WHO's 2009 definition has been widely used for the diagnosis of severe dengue [8,9]. Therefore, we conducted a prospective study among adults admitted to the hospital with dengue in order to determine the clinical factors associated with the development of severe dengue using the WHO's 2009 definition.

\section{Methods}

\section{Study design and population}

This prospective study was conducted at the Hospital for Tropical Diseases, Faculty of Tropical Medicine, Mahidol University in Bangkok, Thailand. Patients admitted to the hospital between October 2012 and December 2014 who met the study criteria were approached for participation. The study's inclusion criteria were: (1) adults at least 15 years old; (2) presenting with clinical criteria for dengue defined as acute fever with at least two of the following symptoms: headache, retro-orbital or ocular pain, myalgia, arthralgia, rash, a positive tourniquet test (defined as the presence of $\geq 20$ petechiae per 1 square inch), or leukopenia (defined as a white blood cell count [WBC] $<5.0 \times 10^{3}$ cells per $\mu \mathrm{L}$ ); and (3) having a confirmed dengue viral infection, defined as positive tests of either (a) viral nucleic acid using reverse-transcriptase polymerase chain reaction (RT-PCR) from serum samples on admission or (b) specific dengue IgM and IgG antibodies using enzyme-linked immunosorbent assays (ELISA) from serum samples on admission and at least two weeks later. Patients with a history of underlying medical illness or mixed infection, or who were pregnant, were excluded from this study.

The laboratory investigations including complete blood count, blood chemistries, and peripheral venous lactate were performed at patient admission. All dengue patients in this study received standard care according to WHO guidelines [8]. Patient data including baseline characteristics, clinical parameters, and laboratory findings were recorded in a pre-defined case-report form. Severity of dengue was summarized on the date of discharge according to the WHO's 2009 definition [8].

\section{Case definition of dengue}

According to the WHO's 2009 dengue case definition [8], dengue patients were classified into non-severe dengue and severe dengue based on clinical and laboratory criteria. Patients with non-severe dengue were subcategorized into two groups depending on the presence or absence of warning signs. Non-severe dengue without warning signs was defined as having acute fever with at least two of the following criteria: nausea, vomiting, rash, myalgia, arthralgia, a positive tourniquet test, or leukopenia. Warning signs included: (1) abdominal pain, (2) persistent vomiting (vomiting with signs of dehydration), (3) clinical fluid accumulation, (4) lethargy, (5) liver span $>15 \mathrm{~cm}$, (6) bleeding from mucosal areas including nose, gums, gastrointestinal tract or vagina, or (7) an elevated hematocrit, $>2 \%$ above the reference range for a healthy Thai adult adjusted for gender with platelet counts $\leq 100 \times 10^{3}$ per $\mu \mathrm{L}$. Severe dengue was classified as having: (1) severe plasma leakage, defined as plasma leakage with shock or respiratory distress (respiratory rate $\geq 24$ breaths/min with oxygen saturation $<95 \%$ in room air and/or requiring oxygen therapy), (2) severe clinical bleeding, defined as spontaneous bleeding from mucosal areas that necessitates a blood transfusion or bleeding in vital organs, (3) severe organ involvement, defined as AST $>1000 \mathrm{IU} / \mathrm{L}$ and/or ALT $>1000$ IU/L, serum creatinine $\geq 3$ times above baseline, myocarditis, and/or encephalitis.

Reverse-transcriptase Polymerase Chain Reaction (RT-PCR) Dengue viral RNA in patient sera was extracted using two rounds of PCR as described by Lanciotti et al. [15] with modifications according to Reynes et al. [16]. Samples were extracted using a PureLink ${ }^{\circ}$ Viral RNA/DNA Mini Kit (Invitrogen ${ }^{\mathrm{m}}$, USA) according to the manufacturer's instructions. 


\section{Serology for dengue viral infection}

All sera were tested with four separate assays of IgM and IgG antibodies for dengue viruses and Japanese encephalitis virus (JEV) using capture ELISA as described by Innis et al. [17]. In order to differentiate dengue viral infections from other flavivirus infections, the ratio of dengue IgM to JEV IgM was calculated. A ratio $\geq 1.0$ was defined as dengue virus infection whereas a ratio $<1.0$ was defined as other flavivirus infection. To assess primary and secondary dengue infections, the ratio of dengue IgM to dengue IgG was calculated. A ratio $\geq 1.8$ was considered a primary dengue infection whereas a ratio $<1.8$ was considered to indicate a secondary dengue infection.

\section{Measurement of peripheral venous lactate}

Blood samples for peripheral venous lactate were collected from veins of upper extremities without the use of a tourniquet. We placed $2 \mathrm{ml}$ of blood in a vacutainer containing sodium fluoride and immediately placed the sample on ice. Samples were sent to the laboratory and lactate levels were tested within 10 min of being drawn using a colorimetric assay (Roche/Hitashi cobas c systems, USA) according to the manufacturer's instructions. The coefficient of variation in lactate assay levels in the central laboratory of the Hospital for Tropical Diseases is estimated to be $1.1 \%$.

\section{Sample-size calculation}

We estimated the required sample size for each potentially associated factor and used the highest estimated number. The required sample size for this study was estimated using the Power and Sample Size Program, version 3.0, 2009 [18]. Based on our previous study, the rate of developing severe dengue among hospitalized adults with dengue who had MAP $\geq 80 \mathrm{mmHg}$ was 0.25 [10]. If the true relative risk of developing severe dengue among patients with MAP $<80 \mathrm{mmHg}$ was double that of patients with $\mathrm{MAP} \geq 80 \mathrm{mmHg}$ then the study required a $1: 2$ ratio of patients with MAP $<80 \mathrm{mmHg}$ to patients MAP $\geq 80 \mathrm{mmHg}$. We needed to study 48 patients with MAP $<80 \mathrm{mmHg}$ and 96 patients with MAP $\geq 80 \mathrm{mmHg}$ to be able to reject the null hypothesis that the relative risk between these groups was equal to 1 with probability (power) of 0.85 . The type I error probability associated with this test was 0.05 . Thus, we needed to study a minimum of 144 patients with dengue.

\section{Statistical analyses}

Data were analyzed using SPSS for Windows 18.0 (IBM Corp., Chicago, IL). Numerical variables were tested for normality using Kolmogorov-Smirnov tests. Variables with non-normal distributions were summarized with medians and inter-quartile ranges (IQR) and compared using Mann-Whitney $U$ tests. Categorical variables were expressed as frequencies and percentages and then analyzed with chi-square tests or Fisher's exact tests, as appropriate. A univariate logistic regression was performed with each potential factor included as an independent variable and the presence or absence of severe dengue infection as the dependent variable. Any variable with a $p$-value less than 0.2 was considered potentially significant and then further analyzed in a stepwise multivariate logistic regression using the backward selection method for determining significant independent factors. All tests of significance were two-sided with $p<0.05$ indicating statistical significance.

\section{Ethics statement}

This study was approved by the Ethics Committee of the Faculty of Tropical Medicine, Mahidol University, Bangkok, Thailand. The procedure indicated by the Standards for the Reporting of Observation Studies in Epidemiology (STROBE) was followed [19]. Written informed consent was obtained from all the patients or the patients' guardians in cases where the patients were less than 18 years of age. Data were made anonymous before analyses.

\section{Results}

From October 2012 to December 2014, there were 236 patients admitted to the Hospital for Tropical Diseases in Bangkok, Thailand, with suspected dengue infections. Of these, 83 patients were excluded from our study because they had a mixed infection (41 patients), a history of underlying medical illness (32 patients), or negative results for dengue in their RT-PCR and IgM/IgG antibody tests (10 patients). Thus, a total of 153 hospitalized patients with confirmed dengue viral infections were recruited to the study. Among these participants, dengue serotypes were identified using RT-PCR in 88 (57.5 \%) patients, indicating DENV 1 (3 patients, $3.4 \%$ ), DENV 2 (9, $10.2 \%)$, DENV 3 (50, $56.8 \%$ ), and DENV 4 (26, $29.5 \%)$. Dengue IgM/IgG antibodies were detected by ELISA in 144 patients (94.1\%) indicating a primary dengue infection in 5 patients $(3.5 \%)$ and secondary dengue infection in 139 patients (96.5\%; see Fig. 1).

Of the 153 patients with a confirmed dengue infection, $132(86.3 \%)$ had non-severe dengue including dengue without warning signs (7 patients, $5.3 \%$ ) and dengue with warning signs (125 patients, $94.7 \%$ ). The remaining 21 patients $(13.7 \%)$ had severe dengue. Of the 21 patients with severe dengue, 16 (76.2 \%) had severe plasma leakage, 16 (76.2 \%) had severe organ involvement, and 8 (38.1\%) had severe clinical bleeding. Of the 16 patients with severe plasma leakage, $12(75.0 \%)$ had plasma leakage with 


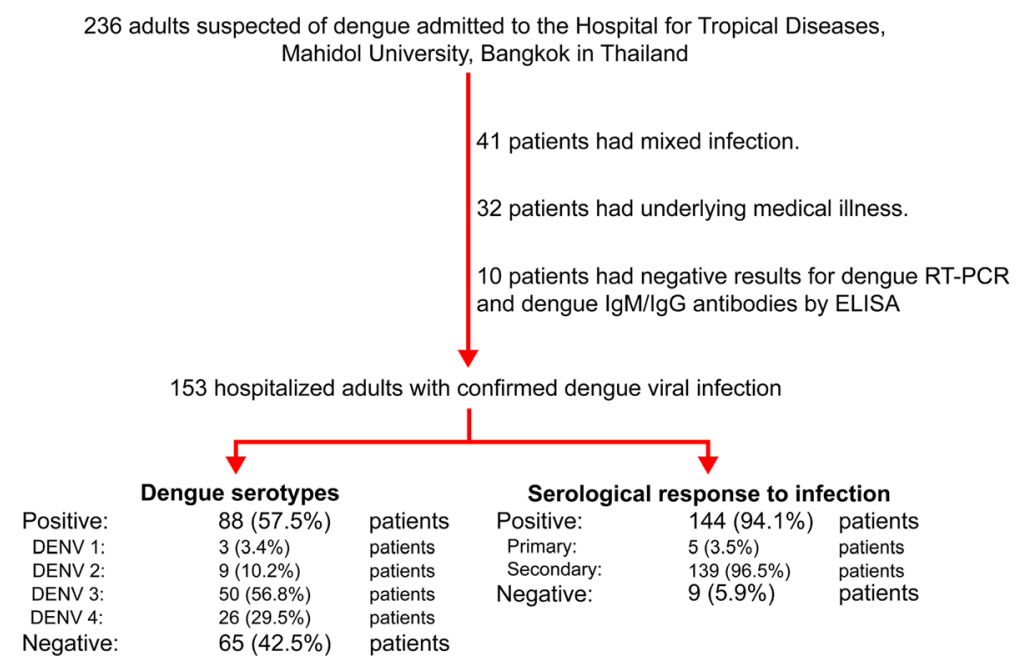

Fig. 1 Flow diagram for recruitment and assessment of study patients

shock and 8 (50.0 \%) had plasma leakage with respiratory distress. Of the 16 patients with severe organ involvement, 9 (56.2 \%) had AST $>1000 \mathrm{IU} / \mathrm{L}$ and/or ALT >1000 IU/L, 9 (56.2 \%) had serum creatinine $\geq 3$ times above baseline, 1 (6.2 \%) had myocarditis, and 1 (6.2\%) had encephalitis.

\section{Comparison between patients with severe and non-severe dengue}

Regarding the baseline characteristics of study patients, the development of severe dengue was significantly associated with (1) patient age being $>40$ years (9 [42.9\%] patients with severe dengue vs. 19 [14.4\%] patients with non-severe dengue; $p=0.004$ ) and (2) patients receiving nonsteroidal anti-inflammatory drugs (NSAIDs) or cyclooxygenase II (COX II) inhibitors before admission (6 [28.6\%] patients with severe dengue vs. 13 [9.8\%] patients with non-severe dengue; $p=0.027$ ). Other patient characteristics such as gender, residential area, and receiving acetaminophen before admission were similar between patients with severe and non-severe dengue (see Table 1).

The majority of symptoms at admission were similar between patients with severe and non-severe dengue with the following exceptions, which were more frequent among patients with severe dengue than those with non-severe dengue (see Table 1): (1) mucosal bleeding (14 [66.7 \%] patients with severe dengue vs. 48 [36.4\%] patients with non-severe dengue; $p=0.017$ ), (2) skin bleeding (13 [61.9\%] patients with severe dengue vs. 28 [21.2 \%] patients with non-severe dengue; $p<0.001)$, (3) liver span $>15 \mathrm{~cm}(12$ [57.1\%] patients with severe dengue vs. 19 [14.4\%] patients with nonsevere dengue; $p<0.001$ ), (4) decreased sound breathing (11 [52.4\%] patients with severe dengue vs. 6 [4.5\%] patients with non-severe dengue, $p<0.001$ ), and (5) persistent vomiting (9 [42.9\%] patients with severe dengue vs. 15 [11.4 \%] patients with non-severe dengue; $p=$ $0.001)$.

Laboratory findings of patients at admission are shown in Table 2. Most laboratory results among patients with severe and non-severe dengue were similar with the exceptions of platelet counts and albumin, which were both significantly lower among patients with severe dengue compared to those with non-severe dengue $(p<0.001)$. However, patients with severe dengue had significantly greater: WBC $(p=0.034)$, absolute atypical lymphocyte count $(p=0.011)$, lactate level $(p<0.001)$, AST level $(p<0.001)$, and ALT level $(p=0.003)$. When patients were categorized based on the upper or lower limits of the reference ranges, patients with severe dengue were significantly more likely to fall into the following categories: absolute lymphocyte count $>2000$ cells per $\mu \mathrm{L}$ $(p=0.036)$, absolute atypical lymphocyte count $>300$ cells per $\mu \mathrm{L}(p=0.002)$, platelet count $\leq 100 \times 10^{3}$ per $\mu \mathrm{L}$ $(p=0.031)$, lactate level $\geq 2.0 \mathrm{mmol} / \mathrm{L}(p<0.001)$, albumin level $<3.5 \mathrm{~g} / \mathrm{dL}(p<0.001)$, AST level $>120 \mathrm{IU} / \mathrm{L}$ $(p=0.017)$, and ALT level $>120 \mathrm{IU} / \mathrm{L}(p=0.032)$. The median (IQR) duration of hospitalization among patients with severe dengue were significantly longer than those with non-severe dengue (4.8 [2.9-9.8] vs. 3.7 [2.7-4.8] days; $p=0.047$ ).

\section{Univariate and multivariate analyses for development of severe dengue}

A univariate logistic regression analysis was used to determine which of the baseline characteristics, clinical parameters, and laboratory findings may be associated with the development of severe dengue. All clinical factors potentially associated with the development 
Table 1 Patient characteristics. Baseline characteristics and clinical parameters at admission of 153 hospitalized adults with either severe or non-severe dengue according to the WHO's 2009 definition

\begin{tabular}{lll}
\hline Characteristics & $\begin{array}{l}\text { Severe dengue } \\
(n=21)\end{array}$ & $\begin{array}{l}\text { Non-severe } \\
\text { dengue }(n=132)\end{array}$ \\
& $\#(\%)$ & $\#(\%)$ \\
\hline
\end{tabular}

Baseline characteristics

Age

$\leq 40$ years

$>40$ years

$12(57.1)$

$113(85.6)$

Gender

Male

Female

Residential area

Bangkok

Outside Bangkok

Medication before

admission

$\begin{array}{lrcr}\text { Acetaminophen } & 20(95.2) & 123(93.2) & 1.000 \\ \text { NSAIDs or COX II inhibitors } & 6(28.6) & 13(9.8) & 0.027 \\ \text { Clinical parameters } & & & \\ \text { Vital signs } & & & \end{array}$

Tempera (IQR), ${ }^{\circ} \mathrm{C}$

\begin{tabular}{|c|c|c|c|}
\hline $\begin{array}{l}\text { HR, median (IQR), beats/ } \\
\text { min }\end{array}$ & $82(68-90)$ & $79(66-88)$ & 0.176 \\
\hline MAP, median (IQR), mmHg & $83(70-92)$ & $86(78-92)$ & 0.561 \\
\hline $\mathrm{PP}$, median (IQR), mmHg & $32(26-45)$ & $38(33-45)$ & 0.058 \\
\hline \multicolumn{4}{|l|}{$\begin{array}{l}\text { History and physical } \\
\text { examinations }\end{array}$} \\
\hline Myalgia & $20(95.2)$ & $120(90.9)$ & 1.000 \\
\hline Lethargy & $19(90.5)$ & $101(76.5)$ & 0.251 \\
\hline Headache & $18(87.5)$ & $113(85.6)$ & 1.000 \\
\hline Fever $\geq 4$ days & $17(81.0)$ & $84(63.6)$ & 0.191 \\
\hline Tourniquet test positive & $16(76.2)$ & $111(84.1)$ & 0.359 \\
\hline $\mathrm{RR} \geq 24$ breaths/min & $15(71.4)$ & $112(84.8)$ & 0.206 \\
\hline Mucosal bleeding & $14(66.7)$ & $48(36.4)$ & 0.017 \\
\hline Skin bleeding & $13(61.9)$ & $28(21.2)$ & $<0.001$ \\
\hline Liver span > $15 \mathrm{~cm}$ & $12(57.1)$ & $19(14.4)$ & $<0.001$ \\
\hline Retro-orbital pain & $11(52.4)$ & $90(68.2)$ & 0.241 \\
\hline Decrease breath sound & $11(52.4)$ & $6(4.5)$ & $<0.001$ \\
\hline Arthralgia & $10(47.6)$ & $37(28.0)$ & 0.120 \\
\hline Rash & $10(47.6)$ & $61(46.2)$ & 1.000 \\
\hline Abdominal pain & $9(42.9)$ & $53(40.2)$ & 1.000 \\
\hline Persistent vomiting & $9(42.9)$ & $15(11.4)$ & 0.001 \\
\hline Diarrhea & $5(23.8)$ & 44 (33.3) & 0.537 \\
\hline
\end{tabular}

Abbreviations: NSAIDs nonsteroidal anti-inflammatory drugs, COX II cyclooxygenase II, IQR interquartile range, $H R$ heart rate, MAP mean arterial pressure, $P P$ pulse pressure, $R R$ respiratory rate of severe dengue were included in the univariate logistic regression analysis. We identified the following clinical factors associated with severe dengue: (1) age $>40$ years, (2) receiving NSAIDs or COX II inhibitors before admission, (3) persistent vomiting, (4) absolute lymphocytes $>2000$ cells per $\mu \mathrm{L}$, (5) absolute atypical lymphocytes $>300$ cells per $\mu \mathrm{L}$, and (6) lactate level $\geq 2.0 \mathrm{mmol} / \mathrm{L}$ (Table 3 ).

All parameters with $p \leq 0.2$ in the univariate logistic regression analysis were then further analyzed by a stepwise multiple logistic regression using the backward selection method in order to determine the independent clinical factors significantly associated with the development of severe dengue. We found the following clinical and laboratory findings to be independently associated with the development of severe dengue: (1) age $>40$ years (odds ratio [OR]: 5.215; CI: $1.538-17.689 ; p=0.008$ ), (2) persistent vomiting (OR: 4.817, CI: 1.375-16.873; $p=0.014)$, (3) absolute atypical lymphocyte $>300$ cells per $\mu \mathrm{L}$ (OR: 3.163, CI: 1.017-9.834; $p=0.047$ ), and (4) lactate level $\geq 2.0 \mathrm{mmol} / \mathrm{L}$ (OR: 7.340, CI: 2.33423.087; $p=0.001$ ) (Table 3).

\section{Lactate level and absolute atypical lymphocytes by dengue severity}

The median (IQR) lactate levels in patients with dengue are shown in Fig. 2. The level of lactate among patients with severe dengue was significantly greater than patients with non-severe dengue $(p<0.05)$ and the extent of elevated lactate was associated with severity of dengue. Similarly, atypical lymphocyte count also increased with severity of dengue (Fig. 3) and patients with severe dengue had significantly higher levels of atypical lymphocytes than those with non-severe dengue with warning signs $(p=0.016)$.

\section{Discussion}

Dengue is the most common mosquito-borne viral disease in humans and it is a significant public health problem, particularly in Asian-Pacific regions like Thailand [1]. Previous reports have shown that both the number of adults infected with dengue and its severity have increased dramatically in recent decades $[2,4-7]$ and the clinical factors associated with severe dengue in adults have varied widely between studies [10-14]. We therefore conducted a prospective study among Thai adults admitted to the hospital with dengue in order to identify the clinical factors associated with severe dengue according to the WHO's 2009 definition, which would help improve the diagnostic process of severe dengue.

The majority of hospitalized adults with dengue had a secondary dengue infection. We found no association between secondary infection and the development of 
Table 2 Laboratory test results. Laboratory parameters on admission of 153 hospitalized adults with dengue by dengue severity according to the WHO's 2009 definition

\begin{tabular}{|c|c|c|c|}
\hline \multirow[t]{2}{*}{ Characteristics } & \multirow{2}{*}{$\begin{array}{l}\text { Severe dengue }(n=21) \\
\#(\%)\end{array}$} & \multirow{2}{*}{$\begin{array}{l}\text { Non-severe dengue }(n=132) \\
\#(\%)\end{array}$} & \multirow[t]{2}{*}{$p$-value } \\
\hline & & & \\
\hline \multicolumn{4}{|l|}{ Confirmation tests for dengue } \\
\hline Known dengue RT-PCR $(n=88)$ & & & 0.765 \\
\hline Serotypes 1 or 4 & $4(19.0)$ & $25(18.9)$ & \\
\hline Serotypes 2 or 3 & $11(52.4)$ & $48(36.4)$ & \\
\hline Known serological response to infection $(n=144)$ & & & 0.551 \\
\hline Primary dengue infection & $1(4.8)$ & $4(3.0)$ & \\
\hline Secondary dengue infection & $20(95.2)$ & $119(90.2)$ & \\
\hline \multicolumn{4}{|l|}{ Complete blood counts } \\
\hline Hemoglobin, median (IQR), g/dL & $14.0(12.5-16.6)$ & $13.8(12.8-14.9)$ & 0.627 \\
\hline Hematocrit, median (IQR), \% & $41.7(36.7-48.5)$ & $41.1(38.3-44.4)$ & 0.934 \\
\hline WBC, median (IQR), $\times 10^{3}$ cells per $\mu \mathrm{L}$ & $4.7(2.4-10.5)$ & $3.1(2.3-4.6)$ & 0.034 \\
\hline Absolute band form, median (IQR), cells per $\mu \mathrm{L}$ & $188(87-372)$ & $142(60-666)$ & 0.237 \\
\hline Absolute PMN, median (IQR), cells per $\mu \mathrm{L}$ & $2320(1210-4126)$ & $1620(873-2264)$ & 0.055 \\
\hline Absolute LYM, median (IQR), cells per $\mu \mathrm{L}$ & $940(405-2011)$ & $758(546-1126)$ & 0.509 \\
\hline Absolute ALYM, median (IQR), cells per $\mu \mathrm{L}$ & $684(108-1370)$ & $138(68-379)$ & 0.011 \\
\hline Platelet counts, median $(\mathrm{IQR}) \times 10^{3}$ per $\mu \mathrm{L}$ & $56.0(12.0-71.0)$ & $85.0(56.0-136.2)$ & $<0.001$ \\
\hline \multicolumn{4}{|l|}{ Categorical data } \\
\hline Hematocrit & & & 0.928 \\
\hline$\leq 2 \%$ above reference range & $7(33.3)$ & $49(37.1)$ & \\
\hline$>2 \%$ above reference range & $14(66.7)$ & $83(62.9)$ & \\
\hline Absolute band form & & & 1.000 \\
\hline$\leq 250$ cells per $\mu \mathrm{L}$ & $15(71.4)$ & $97(73.5)$ & \\
\hline$>250$ cells per $\mu \mathrm{L}$ & $6(28.6)$ & $35(26.5)$ & \\
\hline Absolute PMN & & & 0.422 \\
\hline$<1500$ cells per $\mu \mathrm{L}$ & $7(33.3)$ & $60(45.5)$ & \\
\hline$\geq 1500$ cells per $\mu \mathrm{L}$ & $14(66.7)$ & $72(54.5)$ & \\
\hline Absolute LYM & & & 0.036 \\
\hline$\leq 2000$ cells per $\mu \mathrm{L}$ & $16(76.2)$ & $122(92.4)$ & \\
\hline$>2000$ cells per $\mu \mathrm{L}$ & $5(23.8)$ & $10(7.6)$ & \\
\hline Absolute ALYM & & & 0.002 \\
\hline$\leq 300$ cells per $\mu \mathrm{L}$ & $8(38.1)$ & $99(75.0)$ & \\
\hline$>300$ cells per $\mu \mathrm{L}$ & $13(61.9)$ & $33(25.0)$ & \\
\hline Platelet counts & & & 0.031 \\
\hline$\leq 100 \times 10^{3}$ per $\mu \mathrm{L}$ & $18(85.7)$ & $77(58.3)$ & \\
\hline$>100 \times 10^{3}$ per $\mu \mathrm{L}$ & $3(14.3)$ & $55(41.7)$ & \\
\hline \multicolumn{4}{|l|}{ Blood chemistries } \\
\hline Creatinine, median (IQR), mg/dL & $0.8(0.6-1.1)$ & $0.8(0.6-1.0)$ & 0.739 \\
\hline Lactate level, median (IQR), mmol/L & $2.7(1.7-3.1)$ & $1.4(1.2-1.8)$ & $<0.001$ \\
\hline Albumin, median (IQR), g/dL & $3.7(3.2-4.3)$ & $4.3(4.0-4.5)$ & $<0.001$ \\
\hline AST, median (IQR), IU/L & $219(94-692)$ & $76(37-158)$ & $<0.001$ \\
\hline ALT, median (IQR), IU/L & $100(61-346)$ & $47(18-106)$ & 0.003 \\
\hline
\end{tabular}


Table 2 Laboratory test results. Laboratory parameters on admission of 153 hospitalized adults with dengue by dengue severity according to the WHO's 2009 definition (Continued)

\begin{tabular}{|c|c|c|}
\hline Categorical data & & \\
\hline Lactate level & & \\
\hline$<2.0 \mathrm{mmol} / \mathrm{L}$ & 7 (33.3) & $111(84.1)$ \\
\hline$\geq 2.0 \mathrm{mmol} / \mathrm{L}$ & $14(66.7)$ & $21(15.9)$ \\
\hline Albumin & & \\
\hline$<3.5 \mathrm{~g} / \mathrm{dL}$ & $13(61.9)$ & $128(97.0)$ \\
\hline$\geq 3.5 \mathrm{~g} / \mathrm{dL}$ & $8(38.1)$ & $4(3.0)$ \\
\hline AST & & \\
\hline$\leq 120 \mathrm{IU} / \mathrm{L}$ & $7(33.3)$ & $84(63.6)$ \\
\hline$>120 \mathrm{IU} / \mathrm{L}$ & $14(66.7)$ & $48(36.4)$ \\
\hline ALT & & \\
\hline$\leq 120 \mathrm{IU} / \mathrm{L}$ & $11(52.4)$ & $102(77.3)$ \\
\hline$>120 \mathrm{IU} / \mathrm{L}$ & $10(47.6)$ & $30(22.7)$ \\
\hline
\end{tabular}

Abbreviations: $R T-P C R$ reverse-transcriptase polymerase chain reaction, IQR interquartile range, WBC white blood cell counts, PMN neutrophils, $L Y M$ lymphocytes, $A L Y M$ atypical lymphocytes, AST aspartate aminotranferase, $A L T$ alanine aminotransferase

severe dengue, consistent with previous studies [20, 21]. However, a number of reports showed that the secondary dengue infection was associated with severe dengue $[11,13,22]$. Furthermore, in contrast to previous studies showing that DENV 2 and DENV 3 were associated with the occurrence of severe dengue [23, 24], we found no association between dengue serotypes and disease severity. Indeed, a recent study from Thailand showed that, rather than dengue serotypes, a longer period of time between sequential DENV infections was associated with

Table 3 Regression analyses. Univariate and multivariate logistic regression analysis of baseline characteristic, clinical and laboratory parameters for development of severe dengue according to the WHO's 2009 definition among 153 hospitalized adults with dengue

\begin{tabular}{|c|c|c|c|c|}
\hline \multirow[t]{2}{*}{ Characteristics } & \multicolumn{2}{|c|}{ Univariate logistic regression analysis } & \multicolumn{2}{|c|}{ Multivariate logistic regression analysis } \\
\hline & Odds ratio $(95 \% \mathrm{Cl})$ & $p$-value & Odds ratio $(95 \% \mathrm{Cl})$ & $p$-value \\
\hline \multicolumn{5}{|l|}{ Age } \\
\hline$\leq 40$ years & 1.000 (Reference) & & 1.000 (Reference) & \\
\hline$>40$ years & $4.461(1.655-12.023)$ & 0.003 & $5.215(1.538-17.689)$ & 0.008 \\
\hline \multicolumn{5}{|c|}{ NSAIDs or COX II inhibitors } \\
\hline No & 1.000 (Reference) & & & \\
\hline Yes & $3.662(1.211-11.070)$ & 0.021 & & \\
\hline \multicolumn{5}{|l|}{ Persistent vomiting } \\
\hline No & 1.000 (Reference) & & 1.000 (Reference) & \\
\hline Yes & $5.850(2.114-16.187)$ & 0.001 & $4.817(1.375-16.873)$ & 0.014 \\
\hline \multicolumn{5}{|l|}{ Absolute lymphocytes } \\
\hline$\leq 2000$ cells per $\mu \mathrm{L}$ & 1.000 (Reference) & & & \\
\hline$>2000$ cells per $\mu \mathrm{L}$ & $3.812(1.156-12.574)$ & 0.028 & & \\
\hline \multicolumn{5}{|c|}{ Absolute atypical lymphocytes } \\
\hline$\leq 300$ cells per $\mu \mathrm{L}$ & 1.000 (Reference) & & 1.000 (Reference) & \\
\hline$>300$ cells per $\mu \mathrm{L}$ & $4.875(1.858-12.794)$ & 0.001 & $3.163(1.017-9.834)$ & 0.047 \\
\hline \multicolumn{5}{|l|}{ Lactate level } \\
\hline$<2.0 \mathrm{mmol} / \mathrm{L}$ & 1.000 (Reference) & & 1.000 (Reference) & \\
\hline$\geq 2.0 \mathrm{mmol} / \mathrm{L}$ & $10.571(3.811-29.321)$ & $<0.001$ & $7.340(2.334-23.087)$ & 0.001 \\
\hline
\end{tabular}

Abbreviations: Cl confidence interval, NSAIDs nonsteroidal anti-inflammatory drugs, COX II cyclooxygenase II 


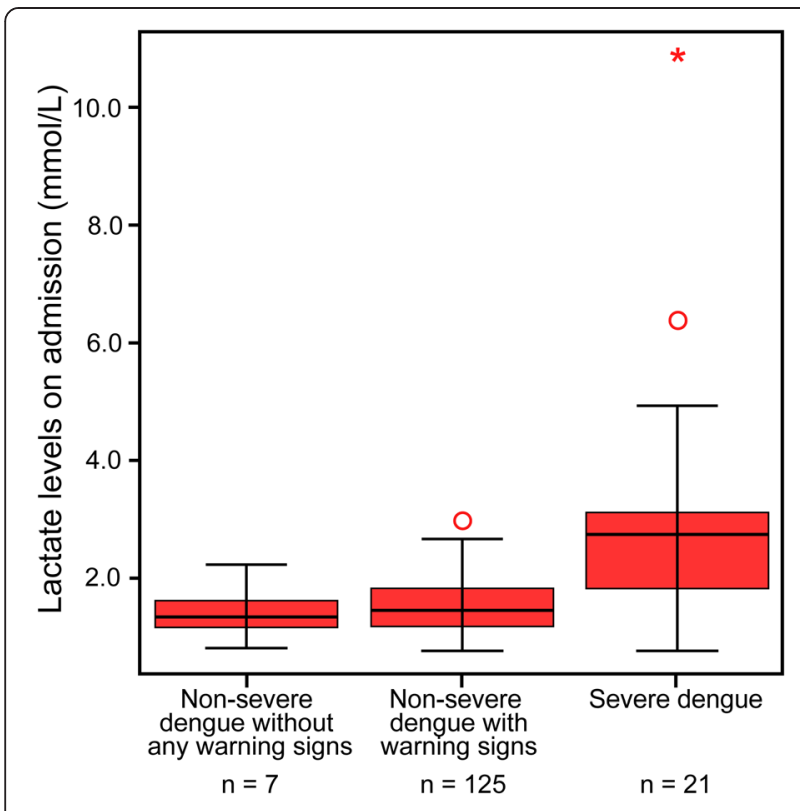

Fig. 2 Lactate levels at admission. Distribution of lactate levels at admission of 153 hospitalized adults with severe dengue and non-severe dengue with and without warning signs (according to the WHO's 2009 definition). Non-severe dengue without warning signs was not significantly different than non-severe with warning signs $(p=0.490)$. Severe dengue was significantly different than both non-severe with warning signs $(p<0.001)$ and non-severe without warning signs $(p=0.006)$

more severe infections, a finding supported by the role of heterotypic immunity in either protection or enhancement of the infection [25].

With regard to the WHO's 2009 guidelines for the management of dengue, patients with warning signs who develop high fever should be administered acetaminophen along with tepid sponge application for reducing the fever; however, NSAIDs should be avoided as these drugs may aggravate the complications, particularly gastrointestinal tract bleeding [8]. At present, it is well established that the use of NSAIDs increase the risk of gastrointestinal, renal, and cardiovascular side effects $[26,27]$. COX II inhibitors have been shown to reduce gastrointestinal side effects, although cardiovascular and renal side effects still develop [27, 28]. A previous report from the Philippines showed that, of 24 American military personnel who were hospitalized with dengue, 1 developed dengue shock syndrome and had upper gastrointestinal bleeding; however, this patient most likely had a history of aspirin use [29]. However, a recent study showed that aspirin could suppress flavivirus replication [30]. Our univariate analysis indicated that patients who received NSAIDs or COX II inhibitors were more likely to develop severe dengue, but that the use of NSAIDs or COX II inhibitors was not independently associated with the development of severe dengue.

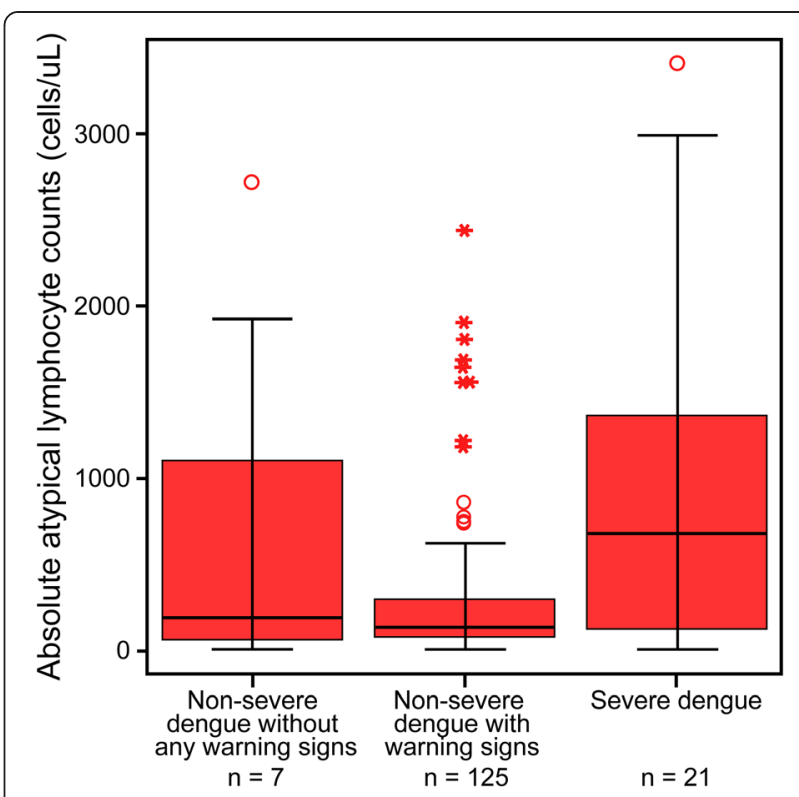

Fig. 3 Absolute atypical lymphocyte levels. Distribution of absolute atypical lymphocytes among 153 hospitalized adults with severe dengue and non-severe dengue with and without warning signs (according to the WHO's 2009 definition). Non-severe dengue without warning signs was not significantly different than non-severe with warning signs $(p=0.859)$. Severe dengue was significantly different than non-severe with warning signs $(p=0.016)$, but not non-severe without warning signs ( $p=0.533)$

In our study, multivariate regression indicated the following clinical and laboratory characteristics upon admission were independently associated with the development of severe dengue: (1) age $>40$ years, (2) persistent vomiting, (3) absolute atypical lymphocyte $>300$ cells per $\mu \mathrm{L}$, and (4) lactate level $\geq 2.0 \mathrm{mmol} / \mathrm{L}$. In addition, patients with severe dengue commonly presented with severe plasma leakage and severe organ involvement. Age $>40$ years was independently associated with severe dengue in our study, consistent with a previous retrospective analysis from France, which reported that plasma leakage was the most common presentation for adults with severe dengue and that an age of $>37$ years predicted plasma leakage [14]. Our results also suggest that persistent vomiting at admission could be used to predict the development of severe dengue, which is similar to a finding of a previous multicenter study showing that persistent vomiting was one of the warning signs for severe dengue [31]. Although the sensitivity of persistent vomiting for identifying severe dengue in adults was very low (6.0-23.0\%), the specificity was as high as $93.0-96.0 \%$ and the negative predictive value was 82.0-97.0\% [32, 33].

Regarding laboratory findings on admission, patients having $>300$ cells $/ \mu \mathrm{L}$ of absolute atypical lymphocytes could be used to predict the development of severe 
dengue as patients with severe dengue had significantly greater levels of absolute atypical lymphocytes than patients with non-severe dengue. These findings are similar to those of a previous Thai study showing that atypical lymphocytes among patients with dengue were correlated with the presence of CD19 + B lymphocytes [34]. After a secondary dengue infection, atypical lymphocytes could indicate an augmented immune response attempting to control the spread of dengue-infected cells [35]. Simultaneously, these antibodies could enhance the entry of the dengue virus into macrophages and dendritic cells whereupon the virus would replicate [36]. Previous reports have also indicated that patients with higher dengue viremia have higher disease severity [37].

In addition, patients having lactate levels $\geq 2.0 \mathrm{mmol} / \mathrm{L}$ could be used as a predictor of the development of severe dengue as elevated lactate levels was observed to correspond to dengue severity in our study. Currently, lactate is used as a biomarker for severity of systemic hypoperfusion or impaired microcirculatory perfusion regardless of organ failure and shock [38]. One previous report has also indicated that elevated arterial or central venous lactate levels could be used to predict in-hospital mortality associated with many conditions such as infection or sepsis, liver disease, trauma, or cardiac arrest [39]. However, the arterial or central venous sampling required to measure lactate concentration is invasive and may increase risk of bleeding in patients with dengue. One previous study has shown a strong correlation between arterial and peripheral venous lactate levels $\left(r^{2}=0.89\right)$ [40], and so we used peripheral venous sampling for lactate concentration. We found that the presence of peripheral venous lactate $\geq 2.0 \mathrm{mmol} / \mathrm{L}$ at admission was independently associated with severe dengue. This finding is similar to that of a previous study among children with dengue [41]. We also show that peripheral venous lactate levels at admission are associated with increasing dengue severity in a dosedependent manner. MAP and pulse pressure at admission were not significantly different among patients with severe and non-severe dengue. Previous studies have suggested that microvascular leakage occurs among patients with severe dengue leading to systemic hypoperfusion or impaired microcirculatory perfusion that can progress to shock $[36,42,43]$. These studies have suggested that systemic hypoperfusion or impaired microcirculatory perfusion in dengue probably occur prior to decreases in blood pressure and other hemodynamic parameters. Another possibility is that blood pressure and pulse pressure are insensitive hemodynamic parameters for identifying systemic hypoperfusion or microcirculatory failure among patients with dengue. In our study, lactate levels at admission could be used as a predictor for the severity of dengue. However, further studies are essential to assess the performance of lactate level as a diagnostic biomarker and/or its combination with other associated clinical factors for identifying severe dengue.

Our study has some limitations: (1) we recruited patients from only a single center in Thailand and our data may not be representative of dengue patients elsewhere, (2) only hospitalized patients were recruited to this study and we included few patients with non-severe dengue without warning signs, and (3) patients were admitted to the hospital at different times following the onset of fever and so the exact time of elevated level of lactate in patients with severe dengue could not be determined.

\section{Conclusions}

Our findings indicate that age ( $>40$ years), persistent vomiting, absolute atypical lymphocytes $(>300$ cells per $\mu \mathrm{L})$, and lactate level $(\geq 2.0 \mathrm{mmol} / \mathrm{L})$ at admission could be used to identify those individuals at greatest risk of developing severe dengue. Early recognition and prompt management of adults with severe dengue might help to reduce morbidity and mortality in future.

\section{Abbreviations}

ALT: Alanine aminotransferase; AST: Aspartate aminotransferase; ALYM: Atypical lymphocytes; Cl: Confidence interval; COX II inhibitors: Cyclooxygenase II inhibitors; DENV: Dengue virus; DHF: Dengue hemorrhagic fever; ELISA: Enzyme-linked immunosorbent assay; HR: Heart rate; IQR: Interquartile range; JEV: Japanese encephalitis virus; LYM: Lymphocytes; MAP: Mean arterial pressure; NSAIDs: Nonsteroidal anti-inflammatory drugs; OR: Odds ratio; PMN: Neutrophils; PP: Pulse pressure; RR: Respiratory rate; RT-PCR: Reverse-transcriptase polymerase chain reaction; STROBE: Standards for the reporting of observation studies in epidemiology; WBC: White blood cell counts; WHO: World health organization.

\section{Competing interests}

The authors declare that they have no competing interests.

\section{Authors' contributions}

VT, NO, VD, and AW conceived and designed of the study. VT, SC, DS, PS, $\mathrm{NO}$, and VD were involved in data collection. VT, NO, DS, and VD performed statistical analyses. AJ and VD performed dengue diagnostic work and provided reagents. VT, VD, and AW wrote the manuscript. VT, NO, and AW performed literature review. All authors read and approved the final manuscript.

\section{Acknowledgements}

We thank all patients who participated in this study. We give special thanks to the staff and nurses in the emergency department and the referral center at Ramathibodi Hospital, Mahidol University, Phramongkutklao Hospital, Rajvithee Hospital, and other private hospitals in Bangkok, Thailand for their contribution in this study. We wish to thank all nurses in private ward 1 and the general female ward at the Hospital for Tropical Diseases, Faculty of Tropical Medicine, Mahidol University for their valuable help in patient care during hospitalization, the staff in the central laboratory at the Hospital for Tropical Diseases, and Ms. Boongomg Noochan in the Clinical Infectious Diseases Research Unit, Department of Clinical Tropical Medicine, Faculty of Tropical Medicine, Mahidol University for help in this study. We also offer sincere thanks to the former Dean, Associate Professor Pratap Singhasivanon, the former Deputy Dean, Associated Professor Porntip Petchmitr, and the Royal College of Physicians of Thailand for their supports in this study. This study was supported by the Dean's fund of the Faculty of Tropical Medicine, Mahidol University, Bangkok, Thailand and by the Royal College of Physicians of Thailand. The funding organization had no role in the study design, conduct of the study, data analysis, or preparation of the manuscript. 


\section{Author details}

'Department of Clinical Tropical Medicine, Faculty of Tropical Medicine, Mahidol University, 420/6 Rajvithi Road, Bangkok 10400, Thailand. ²Pulmonary and Critical Care Division, Department of Medicine, Phramongkutklao Hospital, 315 Rajvithi Road, Bangkok 10400, Thailand. ${ }^{3}$ Information Technology Unit, Faculty of Tropical Medicine, Mahidol University, 420/6 Rajvithi Road, Bangkok 10400, Thailand. ${ }^{4}$ Department of Microbiology and Immunology, Faculty of Tropical Medicine, Mahidol University, 420/6 Rajvith Road, Bangkok 10400, Thailand. ${ }^{5}$ Hospital for Tropical Diseases, Faculty of Tropical Medicine, Mahidol University, 420/6 Rajvithi Road, Bangkok 10400, Thailand

\section{Received: 28 July 2015 Accepted: 26 September 2015} Published online: 14 October 2015

\section{References}

1. World Health Organization (WHO). Global strategy for dengue prevention and control 2012-2020. Geneva: WHO; 2012

2. World Health Organization (WHO). Comprehensive guidelines for prevention and control of dengue and dengue hemorrhagic fever revised and expanded edition. India: WHO; 2011.

3. Ooi EE, Goh KT, Gubler DJ. Dengue prevention and 35 years of vector control in Singapore. Emerg Infect Dis. 2006;12(6):887-93.

4. Wichmann O, Hongsiriwon S, Bowonwatanuwong C, Chotivanich K, Sukthana Y, Pukrittayakamee S. Risk factors and clinical features associated with severe dengue infection in adults and children during the 2001 epidemic in Chonburi, Thailand. Trop Med Int Health. 2004;9(9):1022-9.

5. Kittigul L, Pitakarnjanakul P, Sujirarat D, Siripanichgon K. The differences of clinical manifestations and laboratory findings in children and adults with dengue virus infection. J Clin Virol. 2007:39(2):76-81.

6. Wang CC, Lee IK, Su MC, Lin HI, Huang YC, Liu SF, et al. Differences in clinical and laboratory characteristics and disease severity between children and adults with dengue virus infection in Taiwan, 2002. Trans R Soc Trop Med Hyg. 2009;103(9):871-7.

7. Leo YS, Thein TL, Fisher DA, Low JG, Oh HM, Narayanan RL, et al. Confirmed adult dengue deaths in Singapore: 5-year multi-center retrospective study. BMC Infect Dis. 2011;11:123. doi:10.1186/1471-2334-11-123.

8. World Health Organization (WHO). Guidelines for diagnosis, treatment, prevention and control. Geneva: WHO; 2009.

9. Horstick O, Jaenisch T, Martinez E, Kroeger A, See LL, Farrar J, et al. Comparing the usefulness of the 1997 and 2009 WHO dengue case classification: a systematic literature review. Am J Trop Med Hyg. 2014:91(3):621-34

10. Aung KL, Thanachartwet V, Desakorn V, Chamnanchanunt S, Sahassananda D Chierakul W, et al. Factors associated with severe clinical manifestation of dengue among adults in Thailand. Southeast Asian J Trop Med Public Health. 2013:44:602-12.

11. Malavige GN, Velathanthiri VG, Wijewickrama ES, Fernando S, Jayaratne SD, Aaskov J, et al. Patterns of disease among adults hospitalized with dengue infections. QJM. 2006:99(5):299-305.

12. Lee VJ, Lye DC, Sun Y, Fernandez G, Ong A, Leo YS. Predictive value of simple clinical and laboratory variables for dengue hemorrhagic fever in adults. J Clin Virol. 2008;42(1):34-9.

13. Wichmann O, Gascon J, Schunk M, Puente S, Siikamaki H, Gjørup I, et al. Severe dengue virus infection in travelers: risk factors and laboratory indicators. J Infect Dis. 2007:195(8):1089-96.

14. Thomas L, Brouste $Y$, Najioullah F, Hochedez P, Hatchuel Y, Moravie V, et al Predictors of severe manifestations in a cohort of adult dengue patients. J Clin Virol. 2010;48(2):96-9.

15. Lanciotti RS, Calisher CH, Gubler DJ, Chang GJ, Vorndam AV. Rapid detection and typing of dengue viruses from clinical samples by using reverse transcriptase-polymerase chain reaction. J Clin Microbiol. 1992:30(3):545-51.

16. Reynes JM, Ong S, Mey C, Ngan C, Hoyer S, Sall AA. Improved molecular detection of dengue virus serotype 1 variants. J Clin Microbiol. 2003;41:3864-7.

17. Innis BL, Nisalak A, Nimmannitya S, Kusalerdchariya S, Chongswasdi V, Suntayakorn S, et al. An enzyme-linked immunosorbent assay to characterize dengue infections where dengue and Japanese encephalitis co-circulate. Am J Trop Med Hyg. 1989;40(4):418-27.
18. Dupont WD, Plummer WD. Power and sample size calculations for studies involving linear regression. Control Clin Trials. 1998;19:589-601.

19. von Elm E, Altman DG, Egger M, Pocock SJ, Gøtzsche PC, Vandenbroucke JP, et al. The Strengthening the Reporting of Observational Studies in Epidemiology (STROBE) statement: guidelines for reporting observational studies. Lancet. 2007;370(9596):1453-7.

20. Watts DM, Porter KR, Putvatana P, Vasquez B, Calampa C, Hayes CG, et al. Failure of secondary infection with American genotype dengue 2 to cause dengue haemorrhagic fever. Lancet. 1999;354(9188):1431-4.

21. Olkowski S, Forshey BM, Morrison AC, Rocha C, Vilcarromero S, Halsey ES, et al. Reduced risk of disease during postsecondary dengue virus infections. J Infect Dis. 2013;208(6):1026-33.

22. Guzman MG, Alvarez M, Halstead SB. Secondary infection as a risk for dengue hemorrhagic fever/dengue shock syndrome: an historical perspective and role of antibody-dependent enhancement of infection. Arch Virol. 2013;158(7):1445-59.

23. Nisalak A, Endy TP, Nimmannitya S, Kalayanarooj S, Thisayakorn U, Scott RM, et al. Serotype-specific dengue virus circulation and dengue disease in Bangkok, Thailand from 1973 to 1999. Am J Trop Med Hyg. 2003;68(2):191-202.

24. Libraty DH, Endy TP, Houng HS, Green S, Kalayanarooj S, Suntayakorn S, et al. Differing influences of virus burden and immune activation on disease severity in secondary dengue-3 virus infections. J Infect Dis. 2002;185(9):1213-21.

25. Bhoomiboonchoo P, Nisalak A, Chansatiporn N, Yoon IK, Kalayanarooj S, Thipayamongkolgul $\mathrm{M}$, et al. Sequential dengue virus infections detected in active and passive surveillance programs in Thailand, 1994-2010. BMC Public Health. 2015:15:250. doi:10.1186/s12889-015-1590-z.

26. Scheiman JM, Hindley CE. Strategies to optimize treatment with NSAIDs in patients at risk for gastrointestinal and cardiovascular adverse events. Clin Ther. 2010;32(4):667-77

27. Harirforoosh S, Asghar W, Jamali F. Adverse effects of nonsteroidal antiinflammatory drugs: an update of gastrointestinal, cardiovascular and renal complications. J Pharm Pharm Sci. 2013;16(5):821-47.

28. Dajani EZ, Islam K. Cardiovascular and gastrointestinal toxicity of selective cyclooxygenase-2 inhibitors in man. J Physiol Pharmacol. 2008:59 Suppl 2:117-33.

29. Hayes CG, O'Rourke TF, Fogelman V, Leavengood DD, Crow G, Albersmeyer MM. Dengue fever in American military personnel in the Philippines: clinical observations on hospitalized patients during a 1984 epidemic. Southeast Asian J Trop Med Public Health. 1989;20(1):1-8.

30. Liao CL, Lin YL, Wu BC, Tsao CH, Wang MC, Liu Cl, et al. Salicylates inhibit flavivirus replication independently of blocking nuclear factor kappa B activation. J Virol. 2001:75(17):7828-39.

31. Barniol J, Gaczkowski R, Barbato EV, da Cunha RV, Salgado D, Martínez E, et al. Usefulness and applicability of the revised dengue case classification by disease: multi-centre study in 18 countries. BMC Infect Dis. 2011;11:106. doi:10.1186/1471-2334-11-106.

32. Leo YS, Gan VC, Ng EL, Hao Y, Ng LC, Pok KY, et al. Utility of warning signs in guiding admission and predicting severe disease in adult dengue. BMC Infect Dis. 2013;13:498. doi:10.1186/1471-2334-13-498.

33. Thein TL, Gan VC, Lye DC, Yung CF, Leo YS. Utilities and limitations of the World Health Organization 2009 warning signs for adult dengue severity. PLoS Negl Trop Dis. 2013;7(1):e2023. doi:10.1371/journal.pntd.0002023.

34. Jampangern W, Vongthoung K, Jittmittraphap A, Worapongpaiboon S, Limkittikul K, Chuansumrit A, et al. Characterization of atypical lymphocytes and immunophenotypes of lymphocytes in patients with dengue virus infection. Asian Pac J Allergy Immunol. 2007;25(1):27-36.

35. Carlos CC, Oishi K, Cinco MT, Mapua CA, Inoue S, Cruz DJ, et al. Comparison of clinical features and hematologic abnormalities between dengue fever and dengue hemorrhagic fever among children in the Philippines. Am J Trop Med Hyg. 2005;73(2):435-40

36. Simmons CP, Farrar JJ, Nguyen W, Wills B. Dengue. N Engl J Med. 2012;366(15):1423-32.

37. Vaughn DW, Green S, Kalayanarooj S, Innis BL, Nimmannitya S, Suntayakorn S, et al. Dengue viremia titer, antibody response pattern, and virus serotype correlate with disease severity. J Infect Dis. 2000;181(1):2-9.

38. Mikkelsen ME, Miltiades AN, Gaieski DF, Goyal M, Fuchs BD, Shah CV, et al. Serum lactate is associated with mortality in severe sepsis independent of organ failure and shock. Crit Care Med. 2009;37(5):1670-7.

39. Okorie ON, Dellinger P. Lactate: biomarker and potential therapeutic target. Crit Care Clin. 2011;27(2):299-326. 
40. Gallagher EJ, Rodriguez K, Touger M. Agreement between peripheral venous and arterial lactate levels. Ann Emerg Med. 1997;29(4):479-83.

41. Sirikutt $P$, Kalayanarooj S. Serum lactate and lactate dehydrogenase as parameters for the prediction of dengue severity. J Med Assoc Thai. 2014;97 Suppl 6:S220-31.

42. Bethell DB, Gamble J, Pham PL, Nguyen MD, Tran TH, Ha TH, et al. Noninvasive measurement of microvascular leakage in patients with dengue hemorrhagic fever. Clin Infect Dis. 2001;32(2):243-53.

43. Spronk PE, Zandstra DF, Ince C. Bench-to-bedside review: sepsis is a disease of the microcirculation. Crit Care. 2004;8(6):462-8.

\section{Submit your next manuscript to BioMed Central} and take full advantage of:

- Convenient online submission

- Thorough peer review

- No space constraints or color figure charges

- Immediate publication on acceptance

- Inclusion in PubMed, CAS, Scopus and Google Scholar

- Research which is freely available for redistribution 